\title{
Correlation of Length of Index Finger (2D) to Ring Finger (4D) Ratio (2D:4D) and Coronary Artery Disease - A Study in Male Population of South Rajasthan
}

\section{Nehal Kukda* and SK Verma}

Department of Medicine, Pacific Medical Collage and Hospital, Udaipur, Rajasthan, India

*Corresponding Author: Nehal Kukda, Department of Medicine, Pacific Medical

Collage and Hospital, Udaipur, Rajasthan, India.
Received: June 25, 2021

Published: July 19, 2021

(C) All rights are reserved by Nehal Kukda

and SK Verma.

\begin{abstract}
Human index finger (2D), to ring finger(4D), 2D:4D ratio attract considerable research interest because they exhibit sexual dimorphism and are linked to several traits. The objective of the present study was to find 2D:4D, which could be used as a marker for predisposition to CAD as assessed by ECG, 2D-ECHO and coronary angiography. This study includes 100 male subjects; 50 subjects with CAD and 50 subjects without CAD of age between 30-80 years. Finger lengths were measured twice for both hands using digital calliper. The results were tabulated and subjected to statistical analysis. 2D and 4D ratio was found more in men with CAD which was statistically significant $(\mathrm{P}<0.01)$, supporting a positive correlation between high 2D:4D and CAD in males.
\end{abstract}

Keywords: Finger Length Ratio; 2D:4D; CAD

\section{Introduction}

Cardiovascular diseases (CVD) have become a ubiquitous cause of morbidity and a leading contributor to mortality in most countries. The rise and recent decline of the CVD epidemic in developing countries have been well documented. CVD are conspicuous among world health problems because they account for $16 \%$ of death in developing countries and 48\% in developed countries [1]. The ratio between lengths of $2^{\text {nd }}$ and $4^{\text {th }}$ digit (2D:4D) is negatively related to testosterone. Prenatal levels of testosterone and oestrogen have been implicated in Myocardial Infarction (MI) [2]. It is hypotestosteronaemia which is associated with adverse coronary risk profiles and coronary morbidity and mortality in men, although the relationship is controversial [3]. Testosterone is proved to be protective against MI in men, as men with low 2D:4D are older at first MI than men with high 2D:4D. Recent data suggests that, men with coronary artery disease (CAD) have lower testosterone levels than men with normal angiograms. Among subjects ages 35 to 84 years men have about twice the total incidence or morbid- ity and mortality than that of women [4].2D:4D ratio has been reported to be related to Foetal growth, Hand preference, Autism, Asperger's syndrome, Sperm counts, Family size, Age at MI in men and Breast cancer in women [5]. In addition, 2D:4D in right and left hands has a sexually dimorphic pattern, such that it is more prevalent in males [6]. Also, research reports have emphasized the positive association between 2D:4D ratio in both hands of Chinese men and CAD [7].

There is an increasing body of literature indicating that men with CAD have significantly lower testosterone level than men without CAD [3]. Fink., et al. reported a significant positive correlation between 2D:4D ratio thus suggested a predisposition for men towards CAD as proxy to early sex steroid exposure [8].

A study including 50 men and 50 women in Greece reported that in men with MI, second digit to fourth digit ratios were significantly higher than the respective ratios in healthy men [9]. Ozdogmus., et al. reported a study of 100 heterosexual male autopsies 
found high 2D:4D ratio of right hand which was related to the atherosclerotic plaque formation of right coronary artery [10].

There are limited studies on the correlation of these digit ratio and CAD, in Indian population and that too in South Rajasthan. The present study was undertaken to determine the possible correlation between digit ratio i.e. length of the index finger (2D) and ring finger (4D) ratio; and coronary artery disease in male population of South Rajasthan. Further it was also plan to investigate whether it could be regarded as an indicator to identify CAD at an early age.

\section{Material and Methods}

The study was conducted on 100 male subjects of age between 30 to 80 years. Study group consisted of 50 subjects with coronary artery disease as confirmed from angiography/ECG/ECHO and 50 subjects without coronary artery disease. Study group cases were selected randomly, ECG, 2D-ECHO and coronary angiography (CAG) reports were reviewed to confirm and include patients for the study. For every study case of CAD, a non CAD patient was taken up for the study from the medical OPD.

Subjects with any apparent hand anomalies, inflammation, trauma, deformities, surgery, genetic and neurological diseases were excluded because of their unsuitability for this investigation. The guidelines of institutional ethical committee were followed.

The measurements of second digit (index finger) and fourth digit (ring finger) were taken from the basal crease proximal to the palm to the tip of the finger with the help of sliding digital calliper. Lengths of these fingers were taken in both hands and expressed as $\mathrm{mm}$.

Data compiled and analysis was carried out with relevant standard statistical tests, keeping the null hypothesis as both the ratio of length of fingers and CAD is independent of each other. The relative risk and attributable risk of coronary artery disease was also calculated and presented in the results.

\section{Result and Discussion}

The percentage distribution of CAD and non CAD cases has been plotted graphically in figure 1.

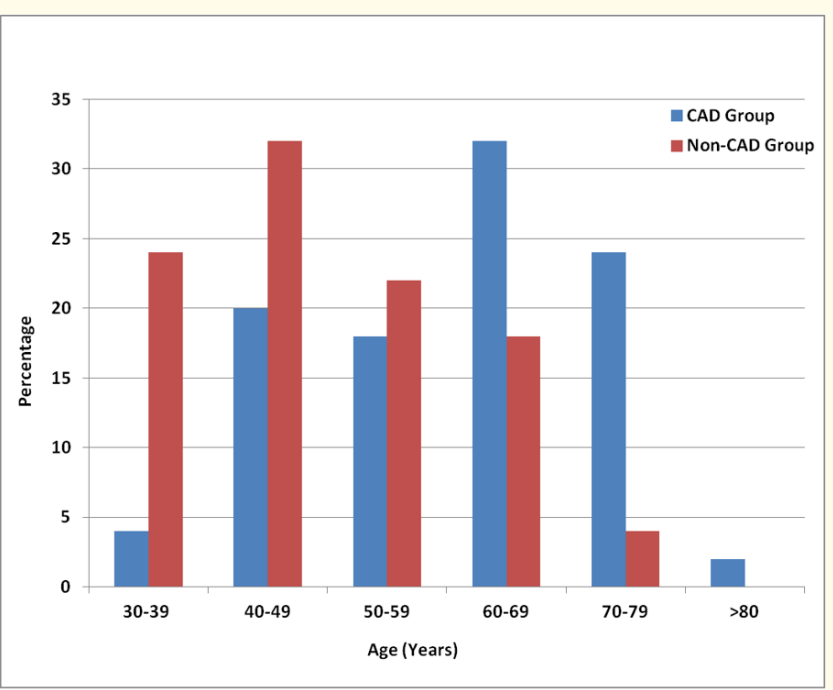

Figure 1: Percentage distribution of CAD and Non-CAD individuals as per age.

Table 1 shows that the mean length of $2 \mathrm{D}$ is 71.5 (SE \pm 0.57 ) in CAD patients whereas it is 72.2 (SE \pm 0.65 ) in Non-CAD patients. Similarly, the mean length of $4 \mathrm{D}$ is 70.7 (SE \pm 0.61 ) in CAD patients whereas it was found to be 69.9 (SE \pm 0.79 ) in Non-CAD patients. Mean ratio of 2D:4D in CAD and Non-CAD patients was $1.005 \pm$ 0.004 and $0.98 \pm 0.003$ respectively and the difference was statistically significant $(\mathrm{P}<0.01)$. However, when mean length of $2 \mathrm{D}$ and $4 \mathrm{D}$ was compared in $\mathrm{CAD}$ and non - CAD group, the difference was statistically not significant $(\mathrm{P}=\mathrm{NS})$.

\begin{tabular}{|c|c|c|c|}
\hline & CAD patients & Non-CAD Individuals & \\
\hline Variables & Mean \pm SE & Mean \pm SE & P value \\
\hline 2D & $71.5 \pm 0.57 \mathrm{~mm}$ & $72.2 \pm 0.65 \mathrm{~mm}$ & NS \\
\hline 4D & $70.7 \pm 0.61 \mathrm{~mm}$ & $69.9 \pm 0.79 \mathrm{~mm}$ & NS \\
\hline 2D:4D & $1.005 \pm 0.004$ & $0.98 \pm 0.003$ & $<0.01$ \\
\hline
\end{tabular}

Table 1: Length of 2D, 4D and ratio of 2D: 4 D in CAD and NonCAD cases.

Interestingly when cases having 2D:4D ratio equal or greater than 1 and less than 1 in CAD and non CAD patients were analysed; 
it was observed that in CAD group 39 (78\%) had 2D:4D ratio more than 1 while $11(22 \%)$ had ratio less than 1 . On the other hand, in non - CAD group, $64 \%$ had ratio less than 1 and only $36 \%$ had 2D:4D ratio greater than 1 (Table 2 ).

\begin{tabular}{|c|c|c|}
\hline Group & CAD (N-50) & Non-CAD (N-50) \\
\hline 2D:4D & $\mathrm{O}=39(78 \%)$ & $\mathrm{O}=18(36 \%)$ \\
Ratio $\geq 1$ & $\mathrm{E}=28.5$ & $\mathrm{E}=28.5$ \\
\hline 2D:4D & $\mathrm{O}=11(22 \%)$ & $\mathrm{O}=32(64 \%)$ \\
Ratio $<1$ & $\mathrm{E}=21.5$ & $\mathrm{E}=21.5$ \\
\hline
\end{tabular}

Table 2: Distribution of cases having 2D:4D ratio greater than or equal to 1 and ratio less than 1 in both CAD and Non-CAD patients. Where $\mathrm{O}$ and $\mathrm{E}$ are observed and expected frequencies for respective cases.

1. $\chi^{2}=17.96, \mathrm{Df}=1, \mathrm{P}<0.01$

2. The Relative Risk of disease $(\mathrm{RR})=2.16$

3. The Attributable Risk of disease (AR) $=53 \%$.

The results were found to be statistically highly significant.

It has been reported that the digit ratio (2D:4D) are the biometric markers that are influenced by testosterone concentration [9]. 2D:4D ratio is prenatally determined and a high 2D:4D ratio has a strong association with lower prenatal androgen [11]. Thus a higher 2D:4D ratio can predict the risk of coronary artery disease at an earlier stage.

In the present study, 2D:4D ratio is significantly higher in males having CAD when compared with the healthy males; a strong correlation of digit ratio to CAD exists as also confirmed through significant chi square test. These findings are similar to the observations reported by other authors $[7,9,12]$. Standard error of difference between the means of 2D and 4D separately did not show significant relation of Coronary Artery Disease to the length of the individual finger.

\section{Conclusion}

The present study concludes that there is an association of high 2D:4D ratio with coronary artery disease. 2D:4D ratio is predictive of cardiovascular diseases and may be used in diagnosis, prognosis and in early life style intervention. Furthermore, it is suggested that similar studies on large number of individuals in different ethnic group of this area may be conducted so as to confirm the association of CAD with 2D:4D ratio.

\section{Author Contribution}

Both the authors have contributed equally to the concept, design and drafting of this manuscript.

\section{Conflict of Interest}

None.

\section{Acknowledgement}

One of the author (Nehal Kukda) extends her sincere thanks to Indian Council of Medical Research (ICMR), New Delhi for financial assistance.

\section{Bibliography}

1. WHO Technical Report Series, No. 1990 (prevention in childhood and youth of adult cardiovascular diseases: time for action: report of a WHO Expert Committee), p. 8.

2. Manning JT and Bundred PE. "The ratio of 2nd and 4th digit length: a predictor of disease predisposition?" Medical Hypothesis 54 (2000): 855.

3. Glueck CJ., et al. "Endogenous testosterone, fibrinolysis and coronary heart disease risk in hyperlipidemic". Journal of Laboratory and Clinical Medicine 122 (1993): 412-420.

4. Lerner DJ and Kannel WB. "Patterns of coronary artery disease morbidity and mortality in the sexes: 26 year follow up of the Framingham Population". American Journal of Cardiology 111 (1986): 383-390

5. Manning JT and Bundred PE. "The ratio of 2nd to 4th digit length and age at first myocardial infarction in men: a link with testosterone?" British Journal of Cardiology 8 (2001): 720-723.

6. Manning JT., et al. "The ratio of 2 nd -4 th digit length: a predictor of sperm numbers and levels of testosterone, LH and oestrogen". Human Reproduction 13 (1998): 3000-3004.

7. Wu XL., et al. "The ratio of second to fourth digit length (2D:4D) and CAD in a Han Chinese population". International Journal of Medical Sciences 10 (2013): 1584-1588.

8. Fink B., et al. "The 2nd-4th digit ratio (2D:4D) and neck circumference: implications for risk factors in coronary artery disease". International Journal of Obesity 30 (2006): 711-4.

9. Kyriakidis I., et al. "Digit ratios and relation to myocardial infarction in Greece men and women". General Medicine 7 (2010): 628-636

10. Ozdogmus 0., et al. "The high 2D:4D length ratio effects on atherosclerotic plaque development". Atherosclerosis 209 (2010): 0195-0196. 
11. Zheng Z and Cohn MJ. "Developmental basis of sexually dimorphic digit ratios". Proceedings of the National Academy of Sciences of the United States of America 108 (2001): 1628916294.

12. Putz DA., et al. "Sex hormones and finger length. What does 2D:4D indicate?" Evolution and Human Behavior 25 (2004): 182-199.

Volume 5 Issue 8 August 2021

(C) All rights are reserved by Nehal Kukda and SK Verma. 